\title{
Recombinant human DNase I decreases biofilm and increases antimicrobial susceptibility in staphylococci
}

\author{
Jeffrey B Kaplan ${ }^{1}$, Karen LoVetri ${ }^{2}$, Silvia T Cardona ${ }^{3}$, Srinivasa Madhyastha ${ }^{2}$, Irina Sadovskaya ${ }^{4}$, \\ Saïd Jabbouri ${ }^{5}$ and Era A Izano ${ }^{1}$
}

Extracellular DNA is an adhesive component of staphylococcal biofilms. The aim of this study was to evaluate the antibiofilm activity of recombinant human DNase I (rhDNase) against Staphylococcus aureus and Staphylococcus epidermidis. Using a 96 -well microtiter plate crystal-violet binding assay, we found that biofilm formation by $S$. aureus was efficiently inhibited by rhDNase at $1-4 \mu \mathrm{gl}^{-1}$, and preformed $S$. aureus biofilms were efficiently detached in $2 \mathrm{~min}$ by rhDNase at $1 \mathrm{mg} \mathrm{I}^{-1}$. Pretreatment of $S$. aureus biofilms for $10 \mathrm{~min}$ with $10 \mathrm{mg} \mathrm{I}^{-1}$ rhDNase increased their sensitivity to biocide killing by 4-5 log units. rhDNase at $10 \mathrm{mg} \mathrm{I}^{-1}$ significantly inhibited biofilm formation by $S$. epidermidis in medium supplemented with sub-MICs of antibiotics. We also found that rhDNase significantly increased the survival of $S$. aureus-infected Caenorhabditis elegans nematodes treated with tobramycin compared with nematodes treated with tobramycin alone. We concluded that rhDNase exhibits potent antibiofilm and antimicrobial-sensitizing activities against $\boldsymbol{S}$. aureus and $\boldsymbol{S}$. epidermidis at clinically achievable concentrations. rhDNase, either alone or in combination with antimicrobial agents, may have applications in treating or preventing staphylococcal biofilm-related infections.

The Journal of Antibiotics (2012) 65, 73-77; doi:10.1038/ja.2011.113; published online 14 December 2011

Keywords: biofilm; dispersin B; DNase; extracellular DNA; Staphylococcus

\section{INTRODUCTION}

Adherent communities of bacteria known as biofilms have a role in the pathogenesis of many chronic infections. ${ }^{1}$ Biofilm bacteria synthesize an extracellular polymeric matrix that holds the cells together and attaches them to the surface. ${ }^{2}$ Extracellular DNA has been shown to be a component of the biofilm matrix produced by many bacteria. ${ }^{3}$ Functions ascribed to extracellular DNA include biofilm cohesion, ${ }^{4}$ cation chelation, ${ }^{5}$ biocide resistance ${ }^{6}$ and genetic exchange. ${ }^{7}$

Several studies have shown that exogenously added DNases can inhibit biofilm formation, detach preformed biofilms and sensitize biofilm bacteria to biocide killing. ${ }^{3}$ Most of these studies utilized laboratory grade DNase I isolated from bovine pancreas. Few studies have measured the antibiofilm activity of pharmaceutical grade recombinant human DNase I (rhDNase). The aim of the present study was to evaluate the effectiveness of rhDNase against biofilmproducing strains of Staphylococcus aureus and Staphylococcus epidermidis. In this report, we present evidence that rhDNase exhibits potent antibiofilm and antimicrobial-sensitizing activities against staphylococci in vitro and in vivo.

\section{MATERIALS AND METHODS}

Reagents

rhDNase (dornase alfa) was obtained from Genentech (South San Francisco, CA, USA). Dispersin B (DspB), a hydrolase that degrades poly- $N$-acetylglucosamine, was purified from a recombinant strain of Escherichia coli as previously described. $^{8}$ Tigecycline was obtained from Pfizer (New York, NY, USA). Povidone iodine was purchased from Ricca (Arlington, TX, USA). Vancomycin, tobramycin, chlorhexidine gluconate and benzalkonium chloride were purchased from Sigma-Aldrich (St Louis, MO, USA).

\section{Bacterial strains and growth conditions}

The S. aureus strains used in this study were $8325,{ }^{9} \mathrm{~N} 315,{ }^{10} \mathrm{SH} 1000,{ }^{11}$ MZ100, ${ }^{12}$ Sa113, ${ }^{13}$ and KBI-11, a clinical isolate (provided by Dr George Zhanel of the University of Manitoba) that did not form significant biofilms in vitro. For S. epidermidis, the biofilm-forming reference strain RP62A (ATCC 35984) was employed. Strains were passaged weekly on blood agar and stored at $4{ }^{\circ} \mathrm{C}$. For in vitro microtiter plate assays, biofilms were cultured in tryptic soy broth (TSB). All media were supplemented with $6 \mathrm{gl}^{-1}$ of yeast extract and $8 \mathrm{gl}^{-1}$ of glucose. All cultures were incubated at $37^{\circ} \mathrm{C}$.

\footnotetext{
${ }^{1}$ Department of Oral Biology, New Jersey Dental School, Newark, NJ, USA; ${ }^{2}$ Kane Biotech, Inc., Winnipeg, Manitoba, Canada; ${ }^{3}$ Department of Microbiology, University of Manitoba, Winnipeg, Manitoba, Canada; ${ }^{4}$ Université Lille Nord de France, Université du Littoral-Côte d'Opale, Boulogne sur mer, France and ${ }^{5}$ Institut de Recherche pour le Developpement, Maadi, Egypt

Correspondence: Dr JB Kaplan, Medical Science Building, Room C-636, 185 S. Orange Ave., Newark, NJ 07103, USA.

E-mail: kaplanjb@umdnj.edu
}

Received 12 May 2011; revised 2 September 2011; accepted 26 September 2011; published online 14 December 2011 


\section{Biofilm formation assay}

Biofilms were cultured in 96-well polystyrene microtiter plates as previously described. ${ }^{6}$ Briefly, cells were diluted to $10^{4}-10^{5} \mathrm{CFU} \mathrm{ml}^{-1}$ in TSB supplemented with antibiotics and enzymes at the indicated concentrations. Aliquots of cells $(200 \mu \mathrm{l}$ each) were transferred to the wells of a microtiter plate, and the plate was incubated at $37^{\circ} \mathrm{C}$ for $18 \mathrm{~h}$. Biofilms were washed with water and then stained for $1 \mathrm{~min}$ with $200 \mu \mathrm{l}$ of Gram's crystal violet. Stained biofilms were rinsed with water and dried. The amount of biofilm biomass was quantified by destaining the biofilms for $10 \mathrm{~min}$ with $200 \mu \mathrm{l}$ of $33 \%$ acetic acid and then measuring the absorbance of the crystal violet solution at $595 \mathrm{~nm}$.

\section{Biofilm killing assay}

Biofilms were grown in 96-well microtiter plates as described above. Biofilms were rinsed with water and then treated with $180 \mu \mathrm{l}$ of TSB supplemented with $10 \mathrm{mgl}^{-1}$ rhDNase. Control wells were treated with $180 \mu \mathrm{l}$ of TSB alone. After $10 \mathrm{~min}$ at $37^{\circ} \mathrm{C}$, a $20-\mu \mathrm{l}$ volume of $4 \%$ chlorhexidine gluconate, $2 \%$ povidone iodine or $0.4 \%$ benzalkonium chloride was added to each well, and biofilms were incubated for $5 \mathrm{~min}$ at room temperature. Control wells received $20 \mu \mathrm{l}$ of water. For biofilms pretreated with TSB alone, biofilms were rinsed four times with PBS to remove the biocide and then treated with $200 \mu \mathrm{lof} 100 \mathrm{mgl}^{-1}$ rhDNase (in $150 \mathrm{~mm} \mathrm{NaCl}$ and $1 \mathrm{~mm} \mathrm{CaCl}_{2}$ ) to dissolve the biofilm. After $10 \mathrm{~min}$, cells were mixed and serial dilutions were plated on agar. For biofilms pretreated with rhDNase, cells were mixed and then quickly diluted 1:1000 in saline $(50 \mu \mathrm{l}$ of cells into $50 \mathrm{ml}$ of saline). The cells were passed through an analytical test filter funnel (no. 145-2020; Nalgene, Rochester, NY, USA), and the filter was then rinsed with $250 \mathrm{ml}$ of sterile water, aseptically removed from the filter unit, and placed on a blood agar plate. Colonies were enumerated after $24 \mathrm{~h}$

\section{Caenorhabditis elegans liquid-medium killing assay}

Infection of C. elegans nematodes with S. aureus was performed as previously described. ${ }^{14}$ Briefly, eggs of C. elegans DH26 (obtained from the Caenorhabditis Genetics Center) were hatched on lawns of E. coli OP50 on nematode growth medium agar. Worms were grown to $\mathrm{L} 4$ stage by incubation at $25^{\circ} \mathrm{C}$ for $48 \mathrm{~h}$. Worms were washed off the plate with M9 buffer, deposited onto lawns of S. aureus KBI-11 prepared according to Sifri et al., ${ }^{15}$ and the plates were incubated at $25^{\circ} \mathrm{C}$ for $17 \mathrm{~h}$. The $S$. aureus-fed worms were washed from the plate with M9 buffer, allowed to settle for $5 \mathrm{~min}$, rinsed with M9 buffer and 1020 worms were transferred to the wells of a 96-well microtiter plate containing $100 \mu \mathrm{l}$ of $80 \% \mathrm{M} 9$ buffer, $20 \%$ TSB with or without $2.5 \mathrm{mgl}^{-1} \mathrm{rhDNase}$ and $1.25 \mathrm{mg} \mathrm{l}^{-1}$ tobramycin. Each treatment was assayed in triplicate. Plates were scored for live worms at the time of inoculation and every $24 \mathrm{~h}$ thereafter for 7 days using a dissecting microscope. Worms were considered dead if they appear straight and alive if they appeared S-shaped and were moving.

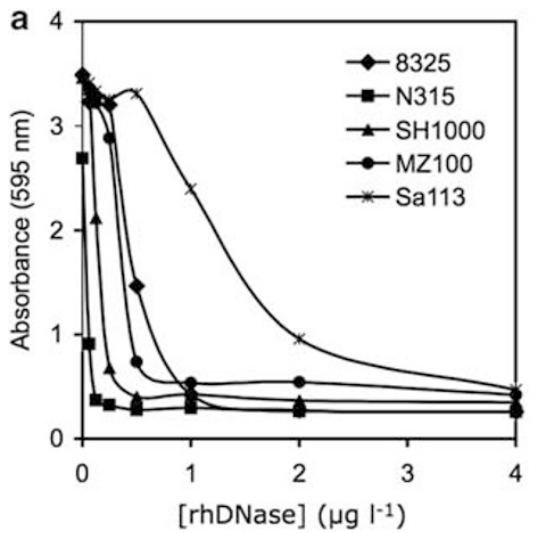

\section{Statistics and reproducibility of results}

All microtiter plate biofilm assays were performed in duplicate wells, which exhibited $<10 \%$ variation in absorbance values. All assays were performed 2-3 times with similarly significant differences in absorbance values. The significance of differences between treatment groups was determined using the Student's $t$ test, with $P$-values of $<0.05$ considered significant. C. elegans infection assays were analyzed using survival curves generated by the Kaplan-Meier statistical method. The log rank test was used to compare survival differences for statistical significance using GraphPad Prism (GraphPad Software, Inc., La Jolla, CA, USA, version 4.0). $P$-values of $<0.01$ were considered significant.

\section{RESULTS}

\section{rhDNase inhibits $S$. aureus biofilm formation}

The ability of rhDNase to inhibit $S$. aureus biofilm formation was measured using a 96-well microtiter plate crystal-violet binding assay (Figure 1a). rhDNase at $4 \mu \mathrm{g} \mathrm{l} \mathrm{l}^{-1}$ efficiently inhibited biofilm formation by five biofilm-forming strains. The concentration of rhDNase required for a $90 \%$ reduction in absorbance values ranged from $0.125 \mu \mathrm{gl}^{-1}$ for strain $\mathrm{N} 315$ to $4 \mu \mathrm{gl}^{-1}$ for strain Sal13.

\section{rhDNase detaches preformed S. aureus biofilms}

The ability of rhDNase to detach preformed $S$. aureus biofilms was measured by culturing biofilms in 96-well microtiter plates and then treating the biofilms with $1 \mathrm{mgl}^{-1}$ rhDNase for increasing amounts of time (Figure 1b). Under these conditions, rhDNase efficiently detached the biofilms produced by all five strains in $2-4 \mathrm{~min}$.

\section{rhDNase sensitizes $S$. aureus biofilms to biocide killing}

We also measured the ability of rhDNase to sensitize preformed S. aureus SH1000 biofilms to killing by the topical biocides chlorhexidine gluconate, povidone iodine and benzalkonium chloride. As shown in Figure 2, biofilms exhibited a 1-log unit reduction in CFU per well values after a 5 -min treatment with chlorhexidine gluconate at $0.4 \%$ or with povidone iodine at $0.2 \%$. Biofilms were resistant to killing by benzalkonium chloride at $0.04 \%$. Treatment of biofilms with $10 \mathrm{mgl}^{-1} \mathrm{rhDNase}$ for $10 \mathrm{~min}$ prior to the addition of the biocides resulted in a $4-5 \log$ unit increase in bacterial killing. rhDNase alone did not exhibit significant antimicrobial activity.

\section{rhDNase enhances tobramycin killing of $S$. aureus in vivo}

The ability of rhDNase to sensitize the $S$. aureus clinical strain KBI-11 to tobramycin killing was measured using a C. elegans in vivo infection

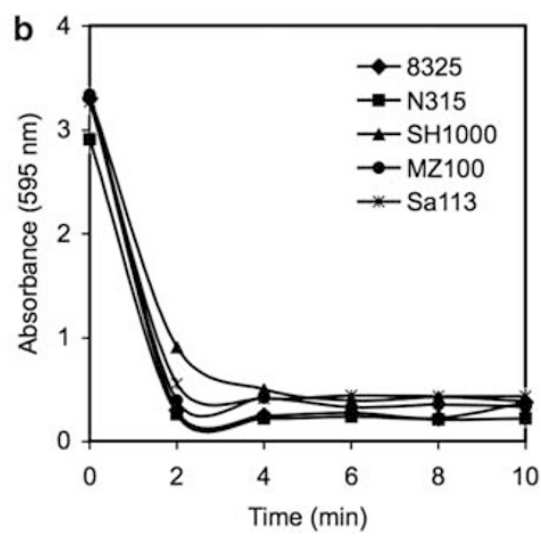

Figure 1 Inhibition (a) and detachment (b) of $S$. aureus biofilms by rhDNase in a 96-well microtiter plate crystal-violet binding assay. (a) The indicated strains were cultured in TSB supplemented with increasing concentrations of rhDNase. Absorbance at $595 \mathrm{~nm}$ is proportional to biofilm biomass. (b) Biofilms were cultured for $18 \mathrm{~h}$ in unsupplemented TSB, rinsed with water and then treated with rhDNase $\left(1 \mathrm{mgl}^{-1}\right)$ for increasing amounts of time. For panels (a) and (b), values represent the means for duplicate wells. Error bars were omitted for clarity. 

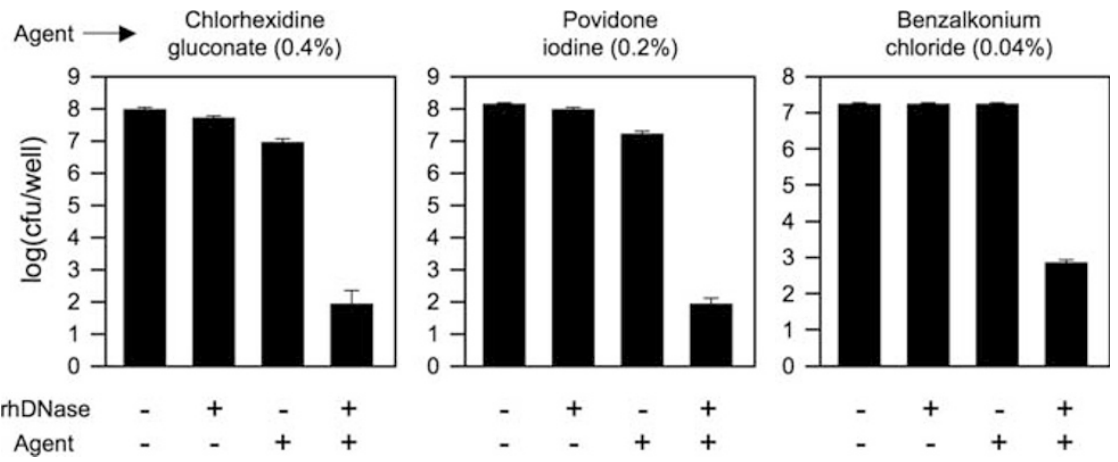

Figure 2 Pretreatment of $S$. aureus biofilms with rhDNase renders them sensitive to killing by topical biocides. S. aureus SH1000 biofilms grown in 96 -well microtiter plates were treated with the indicated agent for $5 \mathrm{~min}$. In some wells, biofilms were pretreated with $10 \mathrm{mgl}^{-1}$ rhDNase for $10 \mathrm{~min}$ prior to the addition of the agent. Values show mean numbers of surviving CFU per well, and error bars indicate range.

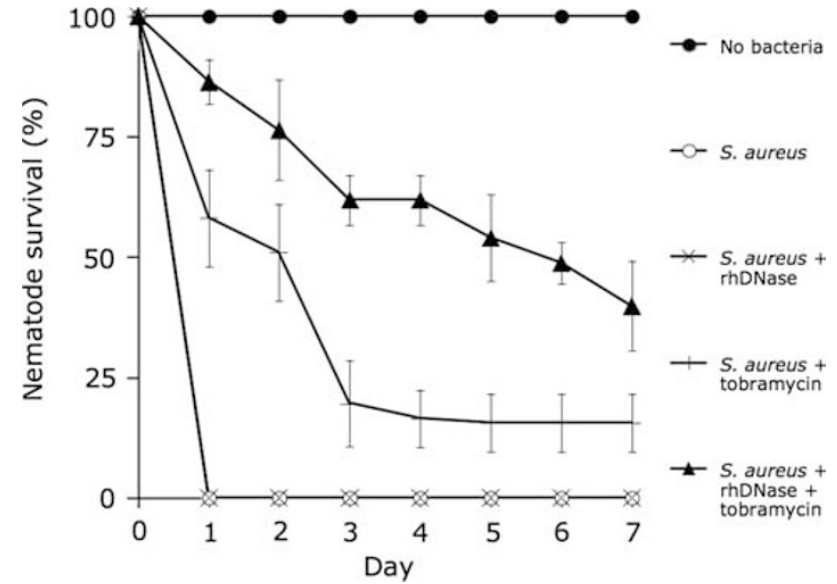

Figure 3 Killing of the nematode $C$. elegans by $S$. aureus in the presence of tobramycin and rhDNase. The graph shows Kaplan-Meier survival curves of uninfected nematodes, nematodes infected with $S$. aureus KBI-11 and nematodes infected with $S$. aureus $\mathrm{KBI}-11$ and cultured in the presence of $2.5 \mathrm{mgl}^{-1}$ rhDNase, $1.25 \mathrm{mgl}^{-1}$ tobramycin, or $2.5 \mathrm{mgl}^{-1} \mathrm{rhDNase}$ plus $1.25 \mathrm{mgl}^{-1}$ tobramycin.

model (Figure 3). Non-mammalian host organisms, including the soil nematode C. elegans, have previously been used to study hostpathogen interactions. Several human pathogens, including Grampositive and Gram-negative bacteria, infect and kill C. elegans when they are supplied as a nutrient source. ${ }^{14,15}$ In this experiment, nematodes were fed $S$. aureus cells for $17 \mathrm{~h}$ and then treated for 7 days with $1.25 \mathrm{mg} \mathrm{l}^{-1}$ tobramycin $(2 \times \mathrm{MIC})$ or $1.25 \mathrm{mgl}^{-1}$ tobramycin plus $2.5 \mathrm{mgl}^{-1}$ rhDNase. Tobramycin significantly increased the survival of nematodes after 1-7 days compared with the no-antibiotic control $(P<0.01)$, and tobramycin plus rhDNase significantly increased the survival of nematodes after 1-7 days compared with tobramycin alone $(P<0.01)$.

\section{rhDNase inhibits $S$. epidermidis biofilm formation}

The ability of rhDNase to inhibit biofilm formation by $S$. epidermidis RP62A, a biofilm-forming reference strain, was measured using a 96well microtiter plate assay (Figure 4). We also measured biofilm inhibition by rhDNase in combination with $\mathrm{DspB}$, a glycoside hydrolase that degrades the major $S$. epidermidis biofilm matrix polysaccharide (poly- $N$-acetylglucosamine). ${ }^{6}$ Antibiotics were added at subMICs to induce biofilm formation. ${ }^{16,17}$ rhDNase significantly inhib-

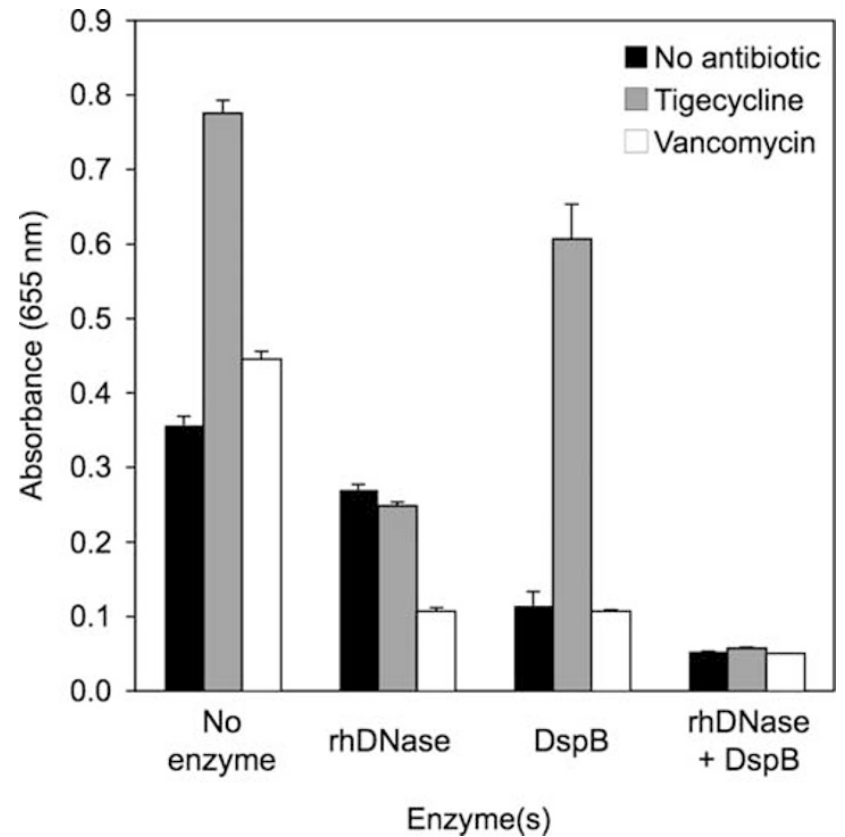

Figure 4 Inhibition of $S$. epidermidis biofilm formation by rhDNase and DspB in 96-well microtiter plates. S. epidermidis strain RP62A was cultured in the presence of $10 \mathrm{mgl}^{-1}$ rhDNase, $10 \mathrm{mgl}^{-1} \mathrm{DspB}$, or a combination of both enzymes at $10 \mathrm{mgl}^{-1}$ each. Some wells were further supplemented with $1.5 \mathrm{mgl}^{-1}$ vancomycin $(2 / 3 \times \mathrm{MIC}), 0.02 \mathrm{mgl}^{-1}$ tigecycline $(1 / 4 \times \mathrm{MIC})$. After $16 \mathrm{~h}$, biofilms were rinsed with water and quantified by crystal violet staining as described in the legend to Figure 1. Values show mean absorbance values for duplicate wells, and error bars indicate range.

ited biofilm formation in the absence or presence of antibiotics $(P<0.05)$. rhDNase was more efficient than DspB at inhibiting $S$. epidermidis biofilm formation in the presence of a sub-MIC of tigecycline. DspB was more effective than rhDNase when tested alone and was comparable to rhDNase plus vancomycin. The combination of rhDNase and DspB inhibited biofilm formation more efficiently than either enzyme alone, both in the absence and presence of antibiotics.

\section{DISCUSSION}

Extracellular DNA has been shown to be an adhesive component of the biofilm matrix synthesized by many different bacteria. ${ }^{3}$ Among the staphylococci, extracellular DNA has been shown to be the major 
matrix component in $S$. aureus biofilms, but only a minor matrix component in S. epidermidis biofilms. ${ }^{6}$

Previous studies showed that various DNases, including bovine DNase I, micrococcal nuclease, restriction endonucleases and human DNase1L2, are capable of inhibiting biofilm formation or detaching preformed biofilms produced by various bacterial species. ${ }^{6,18} \mathrm{Few}$ studies have measured the antibiofilm activity of rhDNase. HallStoodley et al. ${ }^{19}$ showed that a 15 -min treatment with rhDNase at $1 \mathrm{mgl}^{-1}$ caused a $10-73 \%$ decrease in the biomass of Streptococcus pneumoniae biofilms cultured in microtiter plates as determined by COMSTAT analysis. However, rhDNase concentrations of $1 \mathrm{gl}^{-1}$ were required to cause a $67-95 \%$ decrease in biofilm biomass. Parks et al. ${ }^{20}$ showed that a 10 -min treatment with rhDNase at $33 \mathrm{mgl}^{-1}$ caused a $75 \%$ reduction in the biomass of neutrophil-induced 24-h-old Pseudomonas aeruginosa biofilms cultured in microtiter plates as determined by crystal violet staining. However, biofilms grown for $48 \mathrm{~h}$ were reduced by only $42 \%$.

In the present study, we measured the antibiofilm activity of rhDNase against $S$. aureus and $S$. epidermidis. We found that rhDNase at $0.125-4 \mu \mathrm{gl}^{-1}$ efficiently inhibited biofilm formation by $S$. aureus (Figure 1a). Previous studies showed that under conditions identical to those used in the present study, bovine DNase I at $100 \mathrm{mgl}^{-1}$ resulted in $40-50 \%$ inhibition of biofilm formation by $S$. aureus strains SH1000 and MRSA252. ${ }^{6}$ Thus, rhDNase may be a more potent inhibitor of $S$. aureus biofilm formation than bovine DNase I. We also found that rhDNase rapidly and efficiently detached S. aureus biofilms (Figure 1b) and sensitized them to biocide killing (Figure 2) at clinically achievable concentrations. Furthermore, rhDNase significantly increased the survival of $S$. aureus-fed C. elegans nematodes exposed to tobramycin (Figure 3), even with a $S$. aureus strain that did not form biofilms in vitro. These findings underscore the importance of extracellular DNA in S. aureus biofilm cohesion and antimicrobial resistance. $^{6}$

In the present study, we found that rhDNase at $10 \mathrm{mgl}^{-1}$ significantly inhibited biofilm formation by $S$. epidermidis strain RP62A (Figure 4), but the amount of biofilm inhibition was only $25 \%$. These findings are consistent with those of previous studies showing that bovine DNase I concentrations of $2-4 \mathrm{~g} \mathrm{l}^{-1}$ were required to efficiently inhibit biofilm formation by $S$. epidermidis strain $1457 .{ }^{21}$ In the present study, rhDNase was not as effective as DspB at inhibiting S. epidermidis biofilm formation in the absence of antibiotics, but was equally or more effective than DspB at inhibiting biofilm formation in the presence of sub-MICs of tigecycline or vancomycin. These findings suggest that $S$. epidermidis may produce extracellular DNA-dependent biofilms in the response to sub-MICs of antibiotics. ${ }^{16,17}$ Biofilm inhibition was most efficient in the presence of both rhDNase and DspB, supporting the hypothesis that both extracellular DNA and poly- $N$-acetylglucosamine surface polysaccharide contribute to S. epidermidis biofilm cohesion. ${ }^{6}$

In summary, rhDNase efficiently inhibits $S$. aureus and $S$. epidermidis biofilm formation, and disperses preformed $S$. aureus biofilms in vitro. In addition, rhDNase sensitizes $S$. aureus biofilms to biocide killing in vitro and $S$. aureus cells to antibiotic killing in vivo. Furthermore, the combination of rhDNase and $\mathrm{DspB}$ is more efficient than either enzyme alone in inhibiting $S$. epidermidis biofilm formation in the absence or presence of antibiotics in vitro. Thus, rhDNase, either alone or in combination with antimicrobial agents, may have potential applications in developing products for the prevention and treatment of staphylococcal biofilm infections, such as those associated with cystic fibrosis, chronic wounds and medical implants.
One of the main concerns with enzyme-based antibiofilm therapy is that the dispersal of cells from the biofilm colony may increase the risk of bloodstream infections and localized infections at distant sites. Enzyme-based therapies may need to be performed in combination with antimicrobial agents, or their utility may be limited to preventive rather than treatment modalities. In addition, rhDNase may interfere with the structure and function of neutrophil extracellular traps. Another drawback to the use of enzymes is their high cost compared with conventional chemical disinfectants and antibiotics. More studies will be needed to evaluate the potential clinical utility of rhDNase as an anti-infective therapeutic.

\section{CONFLICT OF INTEREST}

JBK receives research funding from Genentech, Inc. This company may develop anti-infective applications based on rhDNase. JBK owns equity in, receives royalty payments from and serves on the advisory board of Kane Biotech, Inc. KL and SM own equity in and are employed by Kane Biotech, Inc. This company is developing antiinfective applications based on DspB. Other authors declare no conflict of interest.

\section{ACKNOWLEDGEMENTS}

We thank Pierre Hardouin and Thierry Grard of the Université du LittoralCôte d'Opale (Boulogne-sur-mer, France), in whose laboratory some of the work was performed. We also thank George Zhanel of the University of Manitoba for providing $S$. aureus strain KBI-11. This study was funded by grants from the US Department of State Fulbright Commission, the Nord-Pas de Calais Regional Council (France), the National Institute of Allergy and Infectious Diseases (award no. AI82392) and Genentech, Inc. (to JBK); and by Manitoba Health Research Council operating grant 309342 (to STC).

1 Donlan, R. M. Biofilms: microbial life on surfaces. Emerg. Infect. Dis. 8, 881-890 (2002).

2 Flemming, H- C. \& Wingender, J. The biofilm matrix. Nat. Rev. Microbiol. 8, 623-633 (2010).

3 Kaplan, J. B. Therapeutic potential of biofilm-dispersing enzymes. Int. J. Artif. Organs 32, 545-554 (2009).

4 Whitchurch, C. B. et al. Extracellular DNA required for bacterial biofilm formation. Science 295, 1487 (2002)

5 Mulcahy, H., Charron-Mazenod, L. \& Lewenza, S. Extracellular DNA chelates cations and induces antibiotic resistance in Pseudomonas aeruginosa biofilms. PLOS Pathog. 4, e1000213 (2009)

6 Izano, E. A. et al. Differential roles of poly- $N$-acetylglucosamine surface polysaccharide and extracellular DNA in Staphylococcus aureus and Staphylococcus epidermidis biofilms. Appl. Environ. Microbiol. 74, 470-476 (2008).

7 Molin, S. \& Tolker-Nielsen, T. Gene transfer occurs with enhanced efficiency in biofilms and induces enhanced stabilisation of the biofilm structure. Curr. Opin. Biotechnol. 14, 255-261 (2003)

8 Yakandawala, N. et al. Enhanced expression of engineered ACA-less $\beta-1,6-N$-acetylglucosaminidase (dispersin B) in Escherichia coli. J. Ind. Microbiol. Biotechnol. 36, 1297-1305 (2009).

9 Novick, R. Properties of a cryptic high-frequency transducing phage in Staphylococcus aureus. Virology 33, 155-166 (1967).

10 Kuroda, M. et al. Whole genome sequencing of meticillin-resistant Staphylococcus aureus. Lancet 357, 1225-1240 (2001)

11 Horsburgh, M. J. et al. $\sigma^{\mathrm{B}}$ modulates virulence determinant expression and stress resistance: characterization of a functional $r s b U$ strain derived from Staphylococcus aureus 8325-4. J. Bacteriol. 184, 5457-5467 (2002).

12 Shanks, R. M. Q. et al. Heparin stimulates Staphylococcus aureus biofilm formation. Infect. Immun. 73, 4596-4606 (2005).

13 Iordanescu, S. \& Surdeanu, M. Two restriction and modification systems in Staphylococcus aureus NCTC8325. J. Gen. Microbiol. 96, 277-281 (1976).

14 Moy, T. I. et al. Identification of novel antimicrobials using a live-animal infection model. Proc. Natl Acad. Sci. USA 103, 10414-10419 (2006).

15 Sifri, C. D. et al. Caenorhabditis elegans as a model host for Staphylococcus aureus pathogenesis. Infect. Immun. 71, 2208-2217 (2003) 
16 Cargill, J. S. \& Upton, M. Low concentrations of vancomycin stimulate biofilm formation in some clinical isolates of Staphylococcus epidermidis. J. Clin. Pathol. 62, 1112-1116 (2009).

17 Rachid, S. et al. Effects of subinhibitory antibiotic concentrations on polysaccharide intercellular adhesin expression in biofilm-forming Staphylococcus epidermidis. Antimicrob. Agents Chemother. 44, 3357-3363 (2000).

18 Eckhart, L. et al. DNase1L2 suppresses biofilm formation by Pseudomonas aeruginosa and Staphylococcus aureus. Br. J. Dermatol. 156, 1342-1345 (2007).
19 Hall-Stoodley, L. et al. Characterization of biofilm matrix, degradation by DNase treatment and evidence of capsule downregulation in Streptococcus pneumoniae clinical isolates. BMC Microbiol. 8, 173 (2008).

20 Parks, Q. M. et al. Neutrophil enhancement of Pseudomonas aeruginosa biofilm development: human F-actin and DNA as targets for therapy. J. Med. Microbiol. 58, 492-502 (2009).

21 Qin, Z. et al. Role of autolysin-mediated DNA release in biofilm formation of Staphylococcus epidermidis. Microbiology 153, 2083-2092 (2007). 